\title{
Fuzzy Logic Based Environment Control of Operation Theatre
}

\author{
Sakshi Srivastav ${ }^{1}$, Ashish Chauhan², Amit Sharma ${ }^{3}$, Ayush Singhal ${ }^{4}$ \\ ${ }^{1}$ Dean Academics, Shri Ram Group of Colleges, Muzaffarnagar, \\ ${ }^{2}$ Department of IT, SRMIST, Ghaziabad \\ ${ }^{3}$ Department of CSE, SRMIST, Ghaziabad, \\ ${ }^{4}$ Department of CSE, Meerut Institute of Technology, Meerut
}

Article History: Received: 10 November 2020; Revised: 12 January 2021; Accepted: 27 January 2021; Published online: 05 April 2021

\begin{abstract}
It is very critical and important to maintain the appropriate climatic conditions in the operation theatre. In paper, we present an approach to control total environmental conditions for the operation theatre. Generally for environmental control of operation theatre air conditioning system is installed. In operation theatre environmental parameters like humidity, temperature, oxygen and particles etc. have to be controlled precisely. All the environmental parameters are of nonlinear nature hence, difficult to control or model with the help of conventional control systems. Keeping in mind the complexity and nonlinearity of these parameters as fuzzy logic controller (FLC) for controlling all the environment of operation theatre has been designed. For this fuzzy logic control system temperature, micro particles, humidity \& oxygen have been taken as input parameters and based on these parameters speed of AC motor as well as the speed of exhaust motor are controlled. The control system is implemented with the help of different fuzzy rules and their membership functions derived from actual conditions. The performance of the system for control of operation theatre was studied and it has been observed that the result obtained with the fuzzy logic control system provides more effective and economical control. The fuzzy logic control system has been implemented using fuzzy Tech development tool.

Keywords: Operation Theatre Monitoring, Fuzzy Logic, oxygen humidity, Temperature Control.
\end{abstract}

\section{Introduction}

A special room designed with completely equipped hospital for performing the surgical operations also known as operation theatre. In 1884 German surgeon Gustav initiate an approach to design an individual operation theatre for the infected and uninfected patient to eliminate the germs with the help of heated and filtered air approach. These Surgical operation theatre is considered as the one of most crucial as well as the complex area in the hospital which is in most careful control of the aseptically conditions of operation theater environment [5]. The main features in this operation that the surgeon mostly observes like the hygiene and the sub elements like heat air and light very carefully. This type of features is not only carries much important conditions of patient health but is necessary to reduce the possibility of complication during surgery and also help for the smooth conduction for the successful operation.

Some uncommon situation containing the temperature in activity theater with specific level, and also decreasing the base level of particles or keeps the idea level for maintaining the dampness level idea for the same and upgrading it into a similar degree of oxygen are vital, henceforth their control is required [6].To implement the environmental conditions (temperature, humidity, particles and oxygen) in operation theatre, generally air conditioning systems are installed. It is well known that the air conditioning systems consisting of mechanical or electrical components are highly nonlinear. The nonlinearities are difficult to realize with conventional (mathematical) model based controller such as (Proportional-Integral-Derivative) PID .So these controllers may not provide the desired environmental conditions of operation theatre.

Fuzzy logic can provide the best control logic under a highly nonlinear system. The FLC can be designed very easily without the complete mathematical knowledge of nonlinear control .FLC are based on linguistic rules such as "IF-THEN" general structure which is the bases of human logic and It is economical also.

In light of the above FLC for controlling the nonlinear environmental conditions of operation theatre has been designed and studied.

For controlling the environment conditions of an operation theatre temperature, humidity, particles and oxygen have been considered as input parameters. Based on the speed of AC motor and exhaust motor parameters can be controlled and these are called output or controlled parameters. The system is implemented with the help of different linguistic rules and their membership functions derived from actual conditions. After implementation it has been observed that with this system the operation theatre environment, fresh air level, humidity, temperature level (at the desired level) can be managed. 
In this research a FLC system has been designed. The benefit of FL is its simplicity. Fuzzy Logic can deal with issues with uncertain and deficient information and it can display nonlinear capacity of self-assertive unpredictability. On the off chance that you don't have a decent plant model or on the off chance that the framework is changing the fuzzy will deliver a superior arrangement, at that point traditional control strategies. For this FLC framework temperature, stickiness, particles and oxygen have been taken as information boundaries; the speed of A C engine and the speed of fumes engine have been controlled as output parameters. The system is implemented with the help of different rules and their membership functions. This work is an attempt to control the optimum conditions required for operation theatre with FLC system.

\section{Method- An Approach}

First we will discuss about the working and designing for air conditioner followed by the working of Fuzzy logic Controller, then we proposed how the working of air conditioner is manages with the help of Fuzzy logic Controller. In Fig 1 describes the components and the functioning of the air conditioning refrigeration cycle.

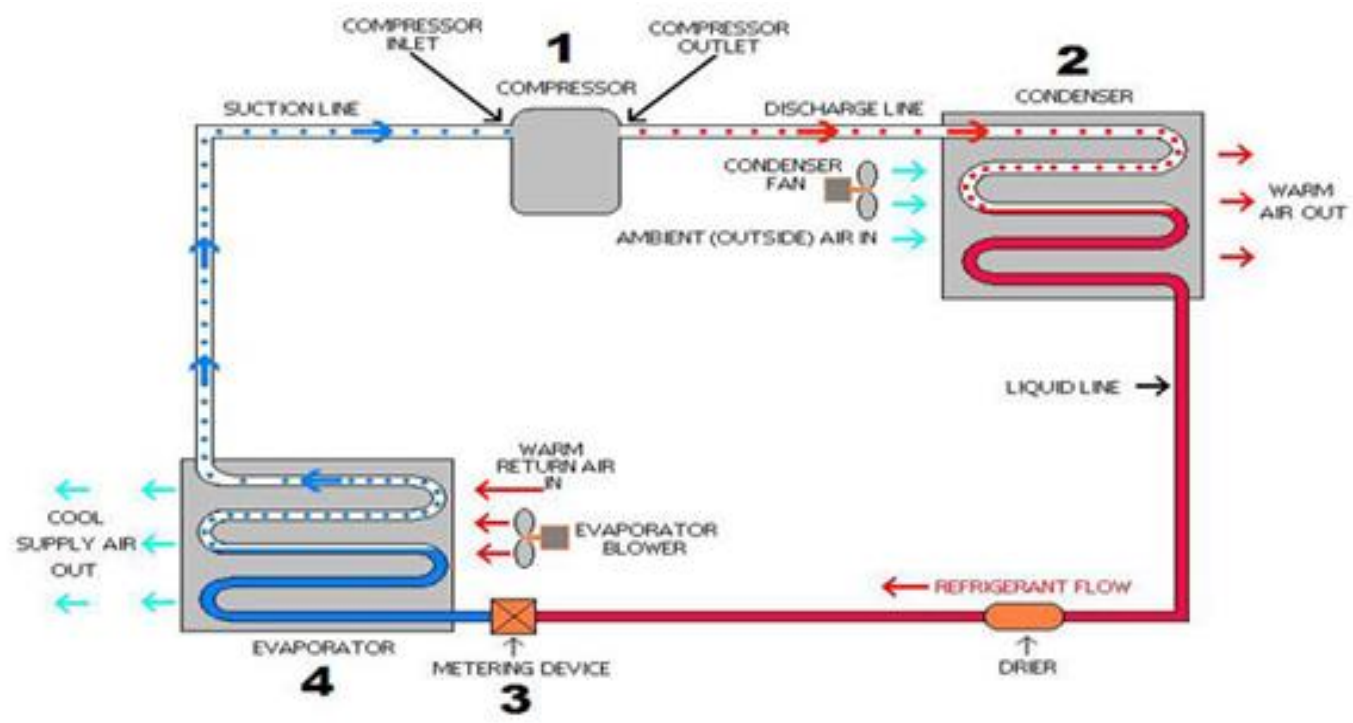

Figure 1. Refrigeration Cycle

The Refrigeration cycle of the airconditioners comprises of the use of chemical that can be easily converted to gas from fluid and back again. The chemical used from the home to the outside air consists of three main parts. These are- Compressor, condenser, evaporator. The condenser and compressor are located on the outside of the air conditioner and the evaporator is located into the home. The compressor is designed to fluid the molecules together and the energy will be high as its temperature. The working liquid leaves the compressor as a hot, high pressure gas and flows into the condenser. In the event that you looked at the air conditioner part outside a house, look for the part that has metal balances all around the blades act simply like a radiator in a car and helps the heat go away, or dissipate, more quickly. At the point when the working liquid leaves the condenser, its temperature is much cooler and it has changed from a gas to a fluid under high pressure. The fluid goes into the evaporator through a exceptionally minuscule, narrowhole. On the other side, the fluid's pressure drops. When it does it starts to evaporate into a gas. As the fluid changes to gas and evaporates, it extracts heat from the air around it. The heat in the air is required to separate the molecules of the liquid from a fluid to a gas. The evaporator also has metal balances to help in exchange the thermal energy with the surrounding air. When the working liquid leaves the evaporator, it is a cool, low pressure gas. It at that point returns to the compressor to start its trip all over again. Connected to the evaporator is a fan that circulates the air inside the housetoblowacross the evaporator balances. Hot air is lighter than cold air, so the hot air in the room raises to the top of a room. There is a vent there where air is sucked into the air conditioner and goes down ducts. The hot air is utilized to cool the gas in the evaporator. As the heat is removed from the air, the air is cooled. It is then blown into the house through other ducts usually at the floor level. This continues over and over and ever until the room reaches the temperature you want the room cooled to. The thermostat faculties that the temperature has reached the correct setting and turns off the air conditioner. As the room warmsup, the thermostat turns the air conditioner back on until the room reaches the temperature. 


\section{Fuzzy Logic Controller- FUZZY LOGIC}

Fuzzy logic means that there are three steps for the system that process appropriate output as required. They may be classified as-
a) Fuzzification
b) Evaluation Rules
c) Defuzzification

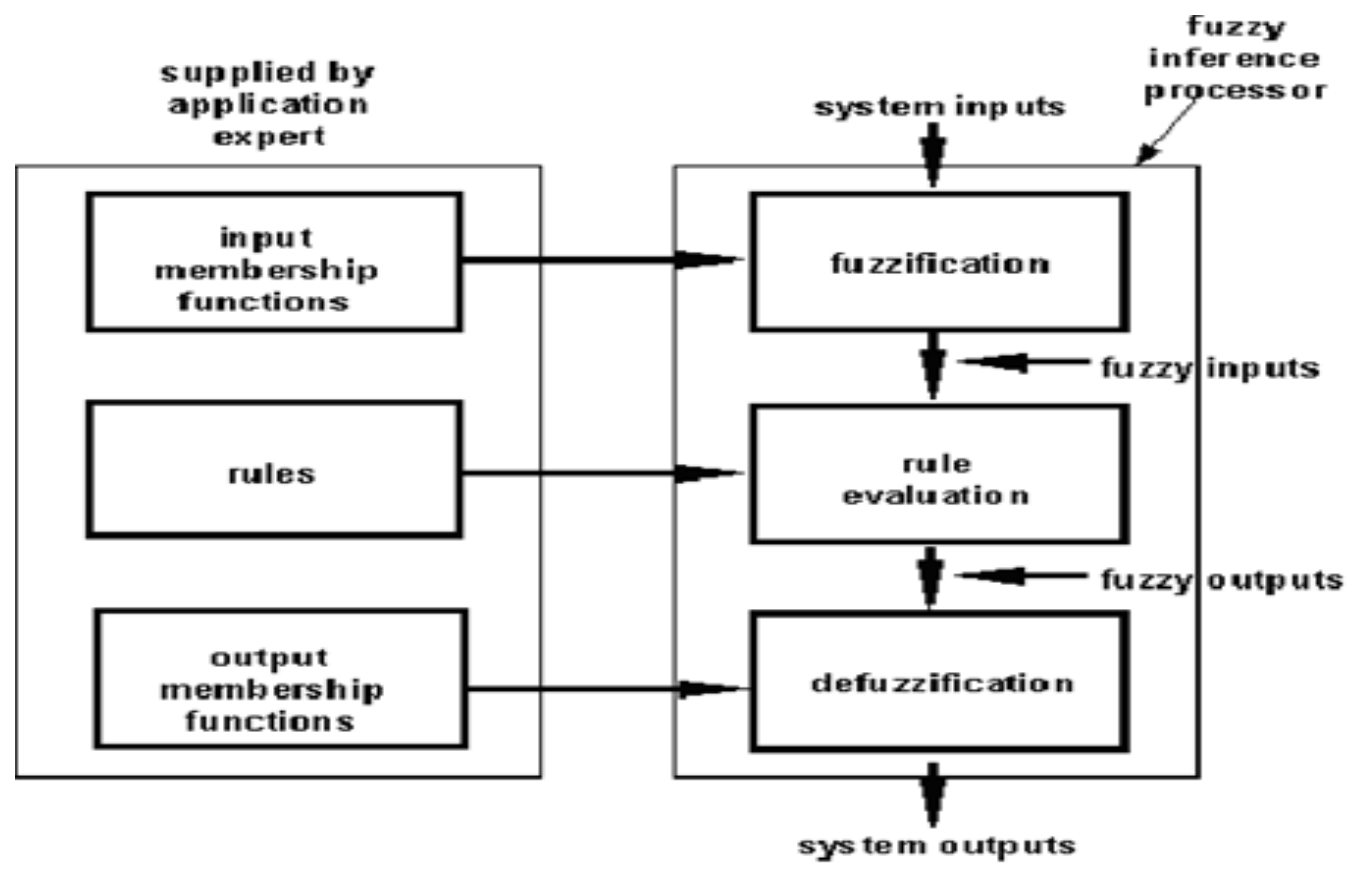

Figure 2. Fuzzy Interference Logic Unit

\section{Fuzzification}

The first step in the fuzzy inference process is the fuzzification where a domain conversion is performed in which crisp inputs given to the system are changed into fluffy data sources. Fresh sources of info are fundamentally the specific data sources read by sensors and passed into the control framework to perform preparing, for example, light, speed, temperature, AQI, pressure, rpm's, and so on Each fresh information that is taken by the framework is then handled by the FIU which contains its own gathering of enrollment capacities or it might have the place that can be changed.

\section{Rule Evaluation}

It is an arrangement of IF-Zadeh operator and THEN standard for deciding the familiarity guidelines as needed for the ideal operation. The former contained the Input label IS and also equal to either the fuzzy input and the truth table required. The output variable of IS label is totally depend upon the Zadeh function that describes the conclusion utilized.

\section{Defuzzification}

In which the output will be in the form of crisp output.

\section{Proposed Model}

The proposed system will assesses the parameters of the air like temperature, mugginess, oxygen and the quantity of particles. Which then all together and as per the estimations of the information boundaries changes the fan's speed of just as the blower engines and the warming and cooling as per the cooling conditions.

On the basis of their values, Three Expression are defined for the input/ output expression as Low, Medium, High.

Input Parameters: HUMIDITY, OXYGEN, TEMPERATURE, PARTICLES. 
Output Parameters : SPEED OF EXHAUST MOTOR, SPEED OF AC MOTOR,

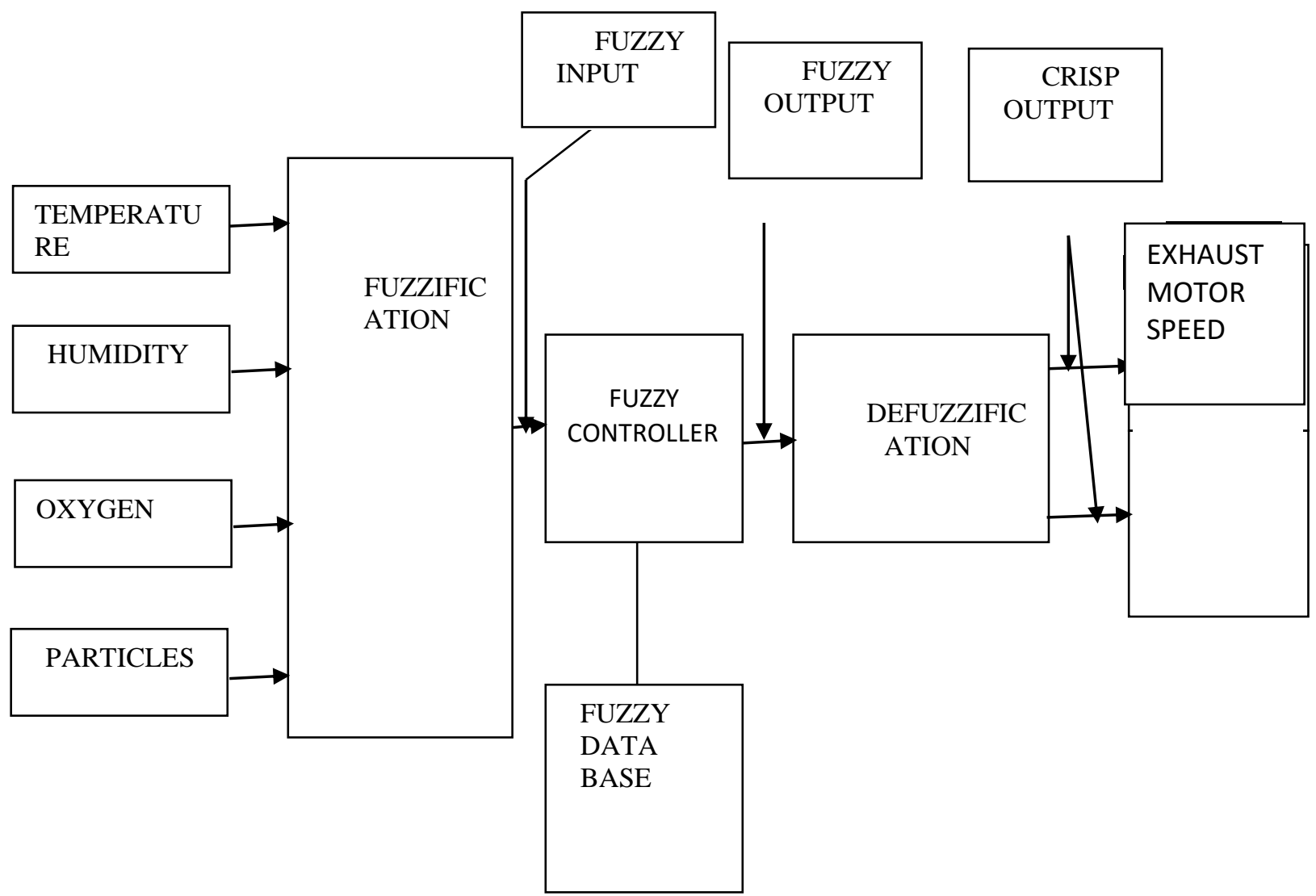

Figure 3. Block Diagram of Proposed Model

Table 1. The input and output Variables / Parameter are shown as-

\begin{tabular}{|l|l|c|c|}
\hline Variables & \multicolumn{1}{|c|}{ Min } & Max & Denomination \\
\hline $\begin{array}{l}\text { Temperature of AC in } \\
\left({ }^{\circ} \mathrm{C}\right)\end{array}$ & 16 & 30 & ${ }^{\circ} \mathrm{C}$ \\
\hline Humidity in (\% & 20 & 70 & $\%$ \\
\hline Oxygen in (\%) & 15 & 2000 & Ppm \\
\hline $\begin{array}{l}\text { Particle (Ppm) } \\
\text { rpm) Motor Speed (in }\end{array}$ & 1000 & 2000 & Rpm \\
\hline $\begin{array}{l}\text { ACxhaust Motor Speed (in } \\
\text { rpm) }\end{array}$ & 1000 & 2000 & Rpm \\
\hline
\end{tabular}

A. Fuzzification of humidity, temperature and particles of oxygen controlling 


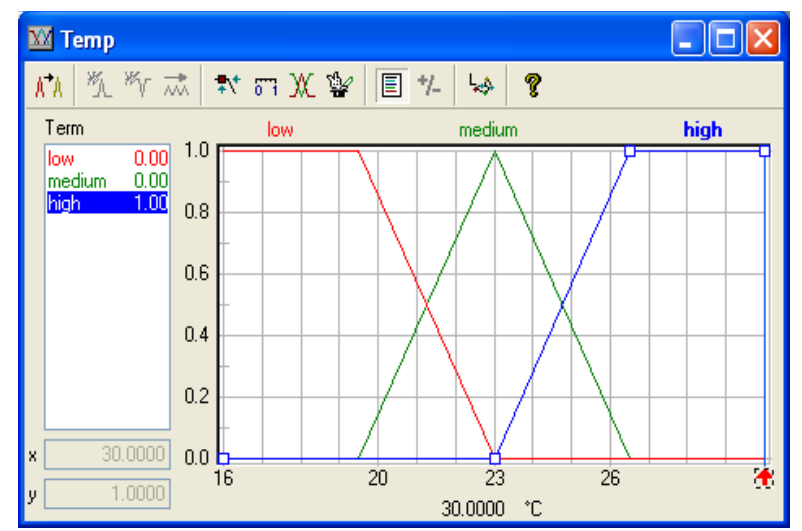

Figure 4.1. Input variable temperature Membership using triangular membership function type

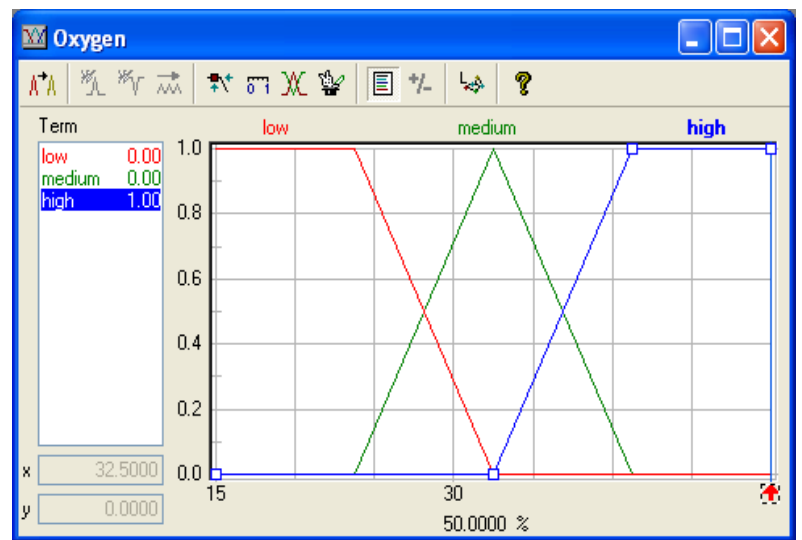

Figure 4.2. Input variable oxygen Membership using triangular membership function type

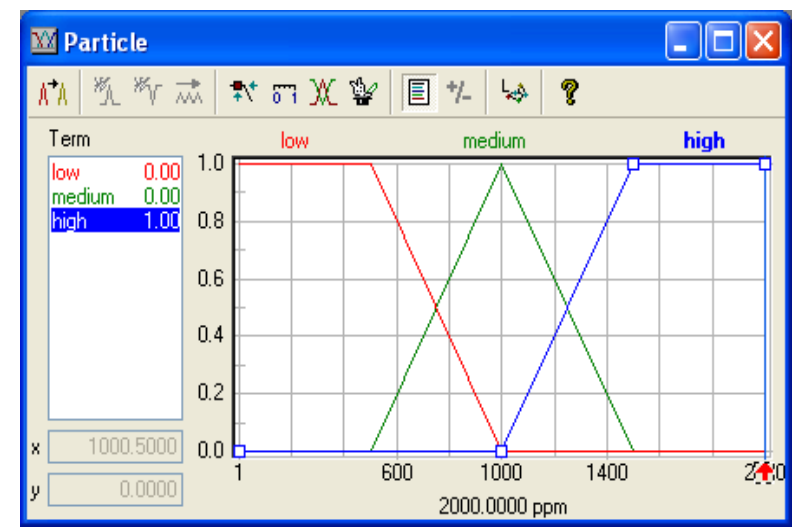

Figure 4.3. Input variable particles Membership using triangular membership function type

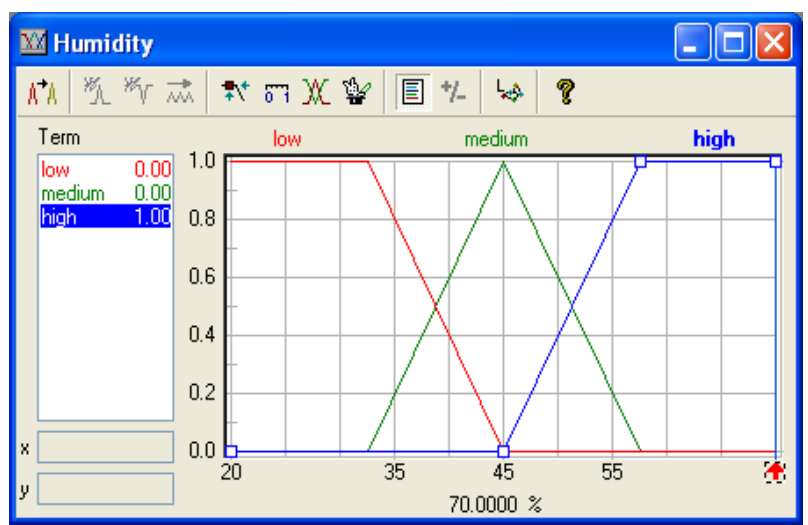

Figure 4.4. Input variable humidity Membership using triangular membership function type 


\section{B. Fuzzy Rule base for air conditioning in operation theatre-}

The rule base decision system of the fuzzy controller is comprised with the set of rules coded in it. These rules are the intuitions of the human beings based upon their experiences and also they are easy to understand. These rule bases are the qualitative statements written in the form of IF-Then sentences. The rule based defined here for the air conditioner is derived from the common sense and are experimentations in a controlled environment.

A simple form of intuitive rules is like:

“IF 'temperature, oxygen, particles, humidity' are high THEN Speed of A C Motor (S1) and Speed of Exhaust Motor (S2) should be high."

Directives that describe the operation theatre for AC controlling as:

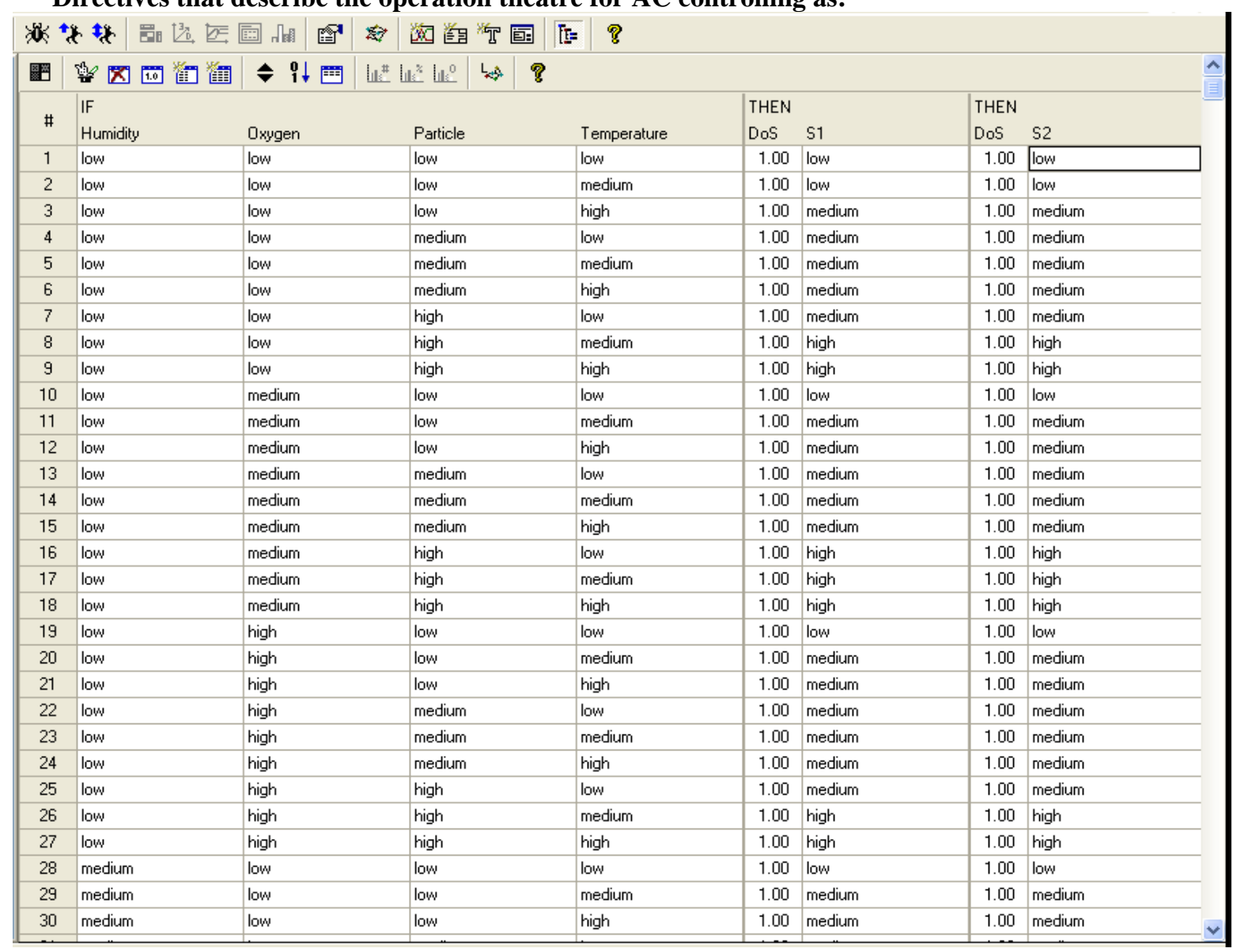




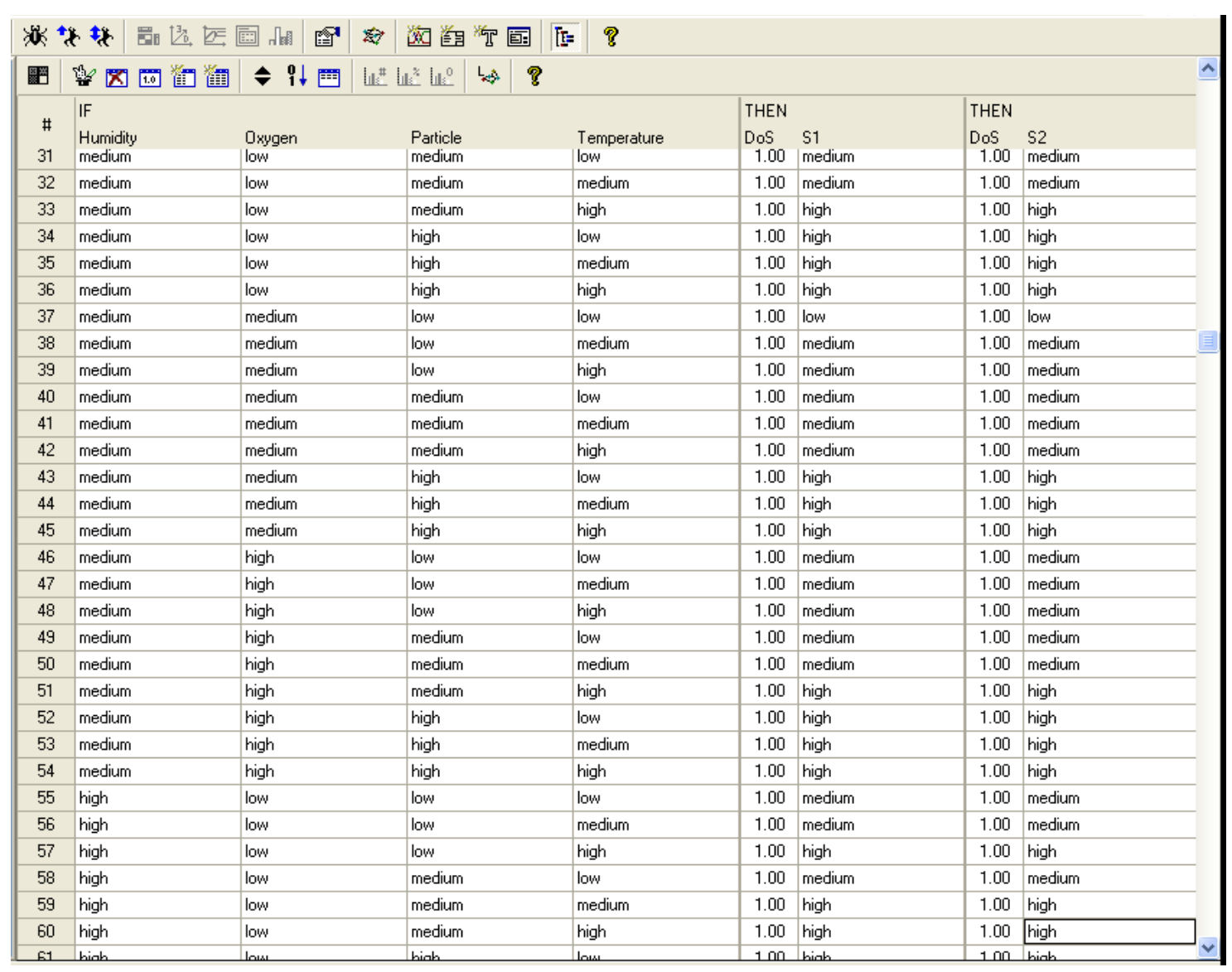

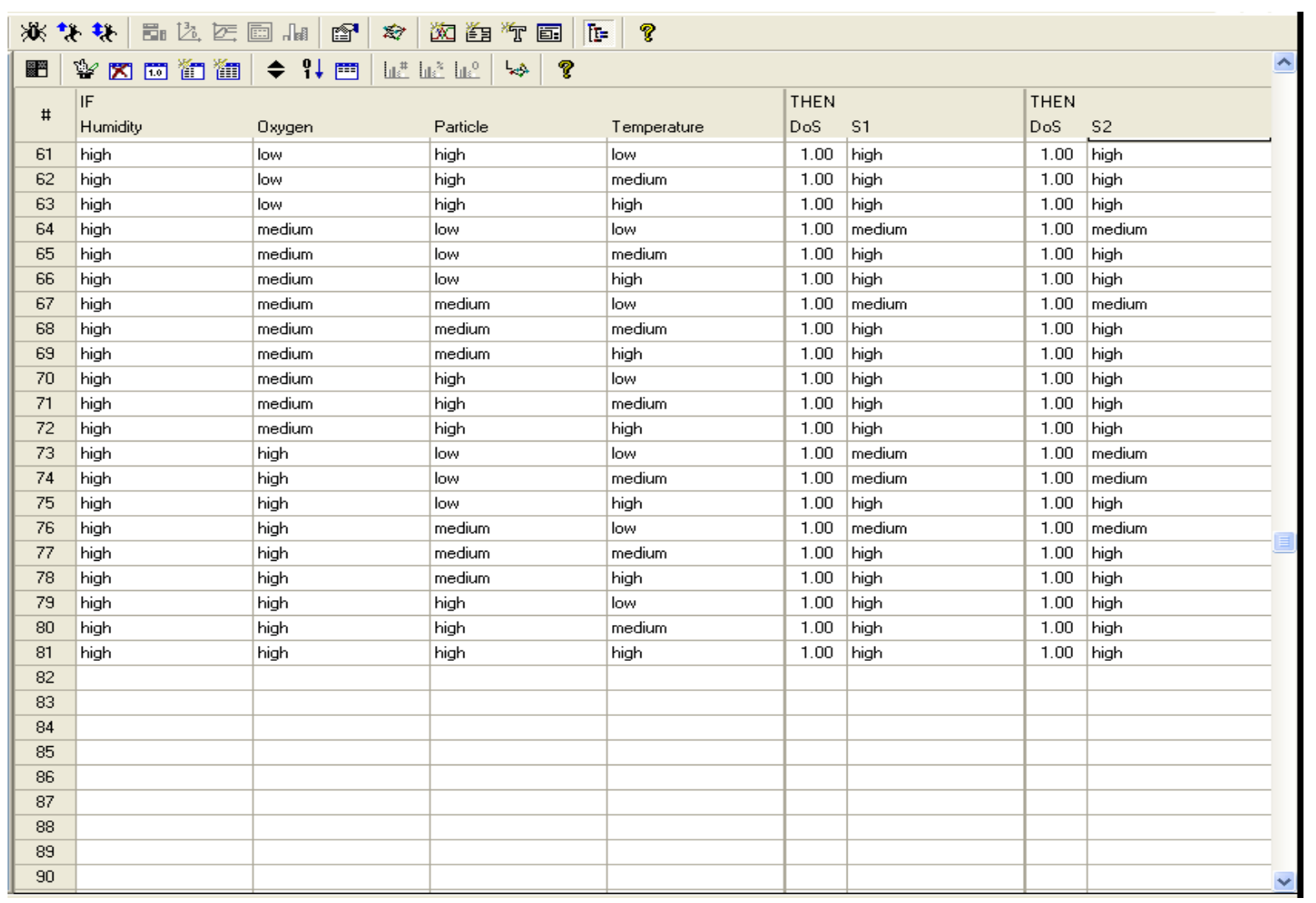

During the findings and result derivation these rules are applied for the method descired for the output produced. 


\section{Result And Discussion}

This research shows the simulation consequences of fuzzy rationale control that was mimicked utilizing Fuzzy technology and after effect of fuzzy rationale control that actualized on two yield boundaries portrayed as S1 and S2. The outcome shows the yield of fluffy rationale that executed on two yield boundaries of fluffy logic. Although there is a distinction in a few yield esteem, however the capacity to control the forced air system is effectively planned as per the ideal.

Figure below show the control of air conditioning in Operation Theatre.

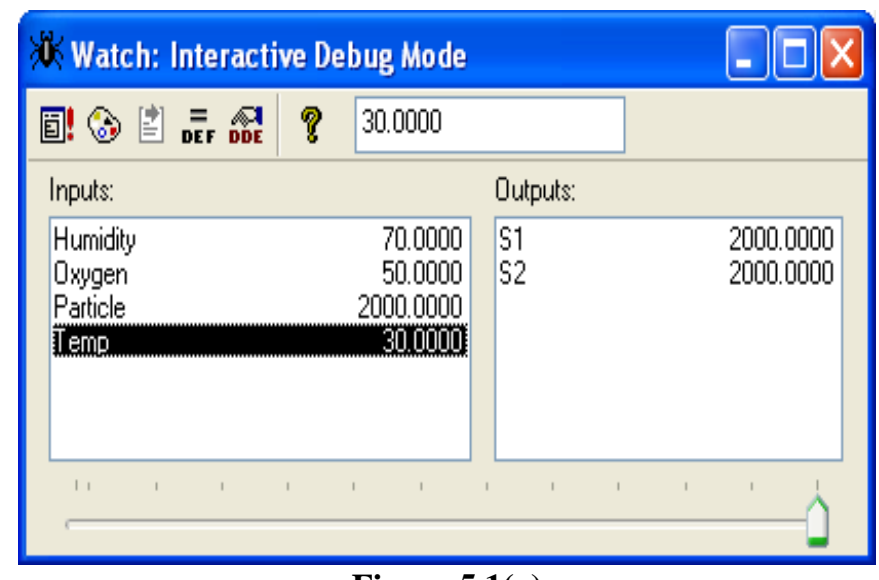

Figure 5.1(a)

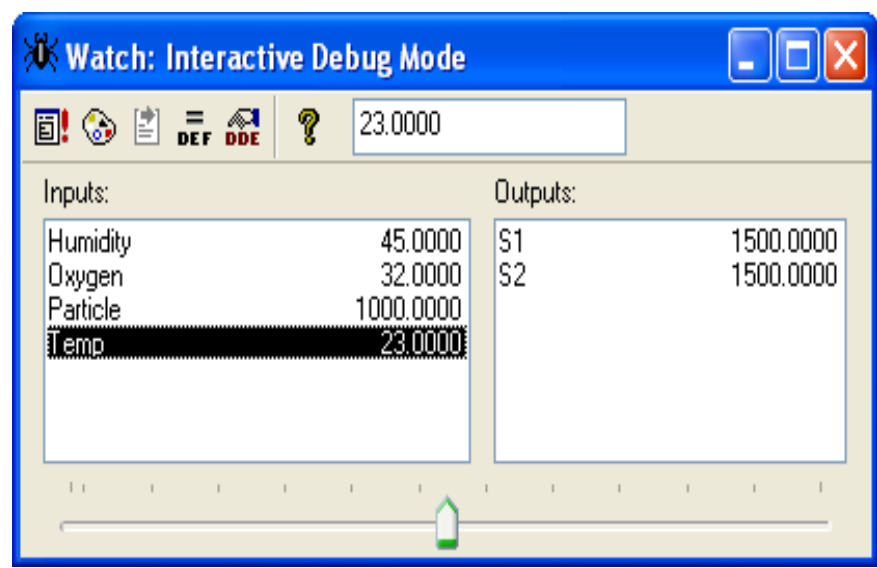

Figure 5.1(b)

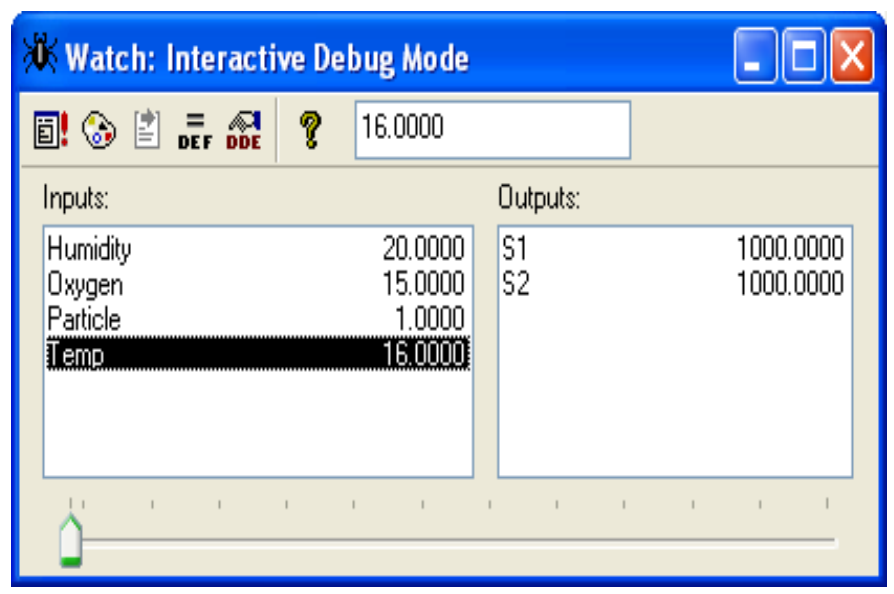

Figure 5.1(c) 


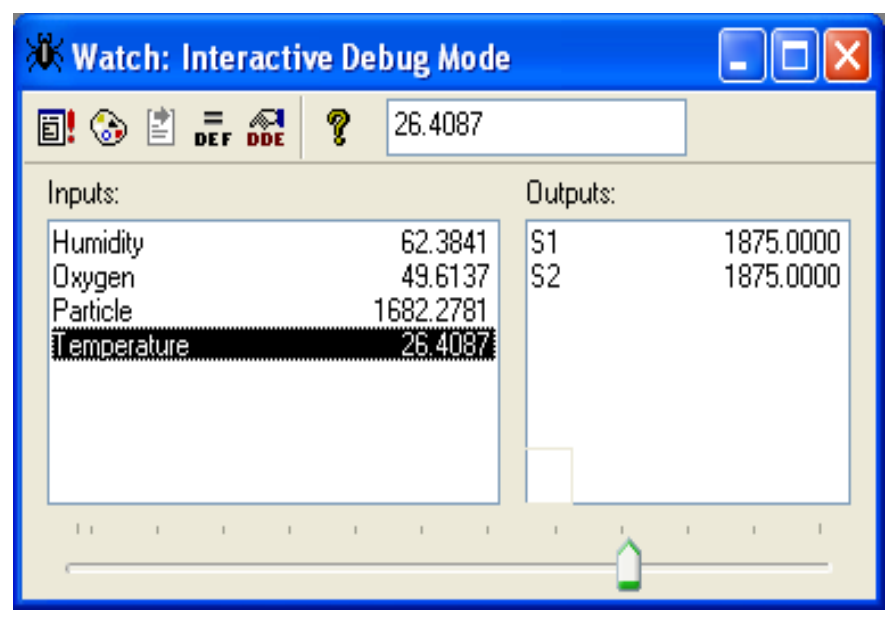

Figure 5.1(d)

These figure shows that (a) when the value of input parameters i.e. is too particle, humidity, temperature, oxygen but the fumes and AC engine speed much maintain at significant level. (b) On lessening the estimation of info boundaries for example moistness, temperature, particle, humidity, temperature, oxygen but the fumes and AC engine speed is likewise diminished to medium. (c) Again on more decrease in the estimation of boundaries esteem for example mugginess, temperature, oxygen but the fumes and AC engine is additionally at low level. (d) When the estimation of temperature is $26.4087 \mathrm{C}$ and at the dampness level $62.3841 \%$, the estimation of particles at $1682.2781 \mathrm{ppm}$ and at the oxygen level $49.6137 \%$; the Fumes and $\mathrm{AC}$ engine speed will be encountered as $1875 \mathrm{rpm}$.

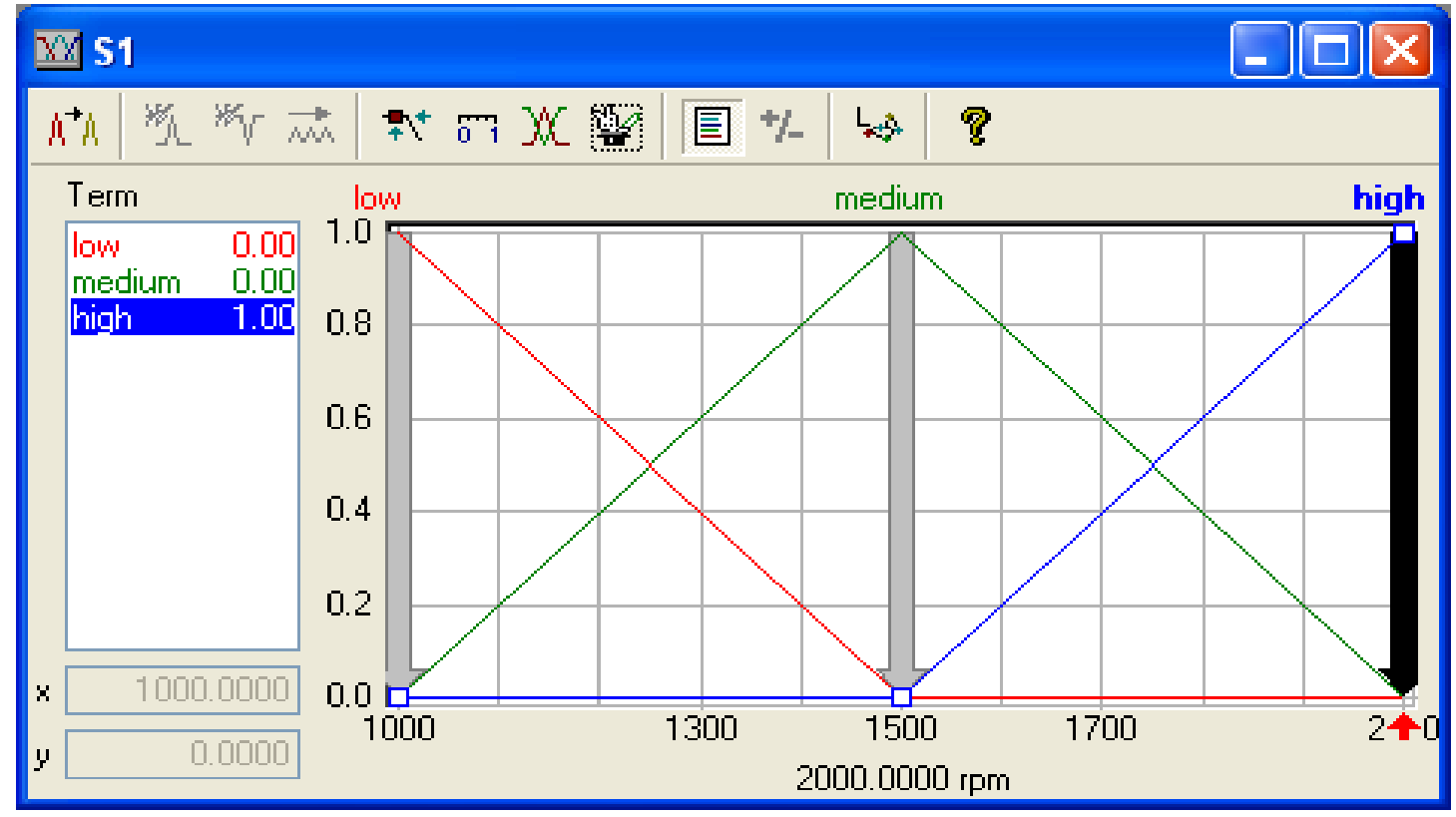

Figure 6. Function of output membership controlling the temperature, humidity, oxygen, particles

Above figure display their yield factors with their related enrollment capacities. Enrollment for the variable speed and plot the function for the AC engine S1/Speed of Exhaust engine S2 utilizing three-sided participation type of work. Finally, in this figure there are three participation of work capabilities are used as Low, Medium, high. 


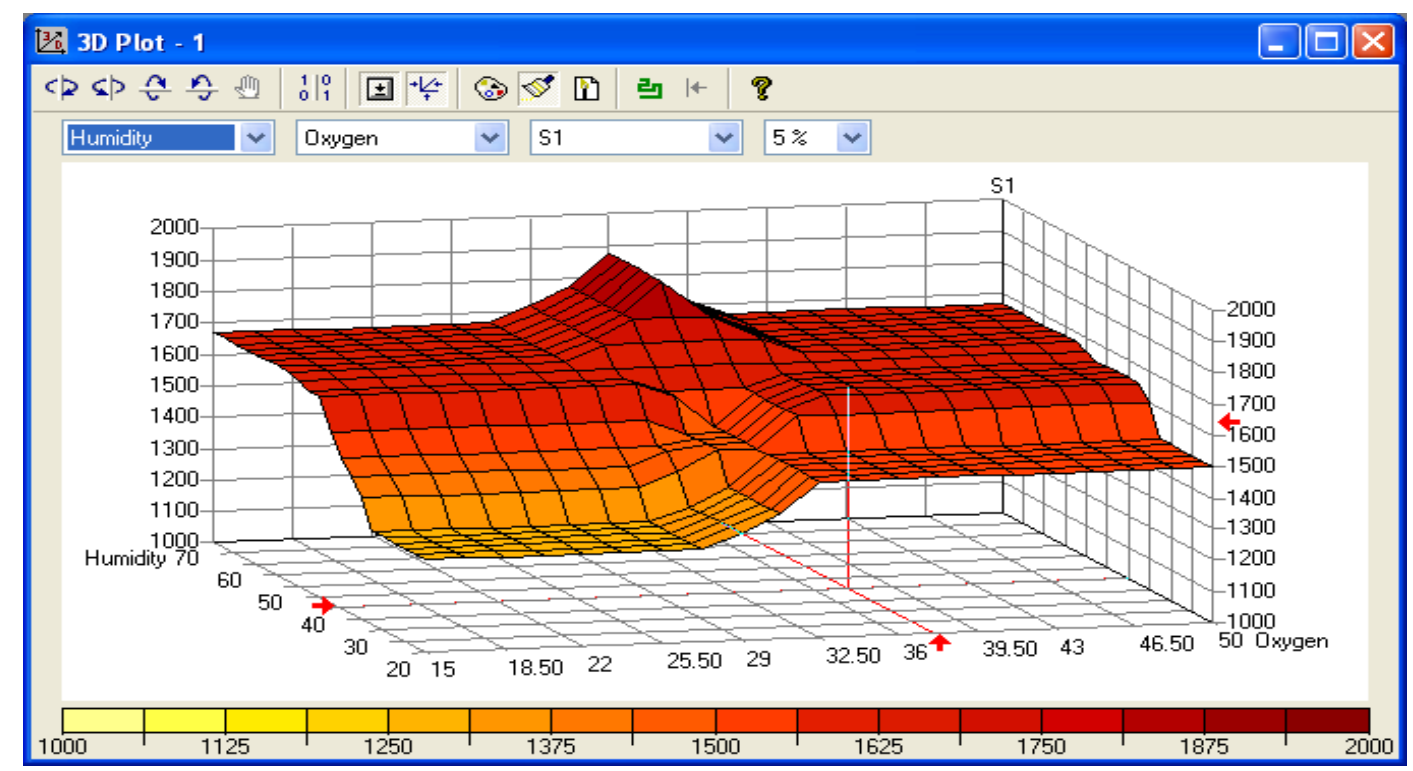

Figure 7. 1 3-D plots for oxygen, humidity \& S1/S2

The above figure plot show that when the value of oxygen and humidity increases, the value of output parameter S1/S2 also raised. As per the above figure the humidity will be countered as $40 \%$ and the oxygen values will be $37 \%$ and their speed is $1632.8300 \mathrm{rpm}$ for $\mathrm{S} 1 / \mathrm{S} 2$.

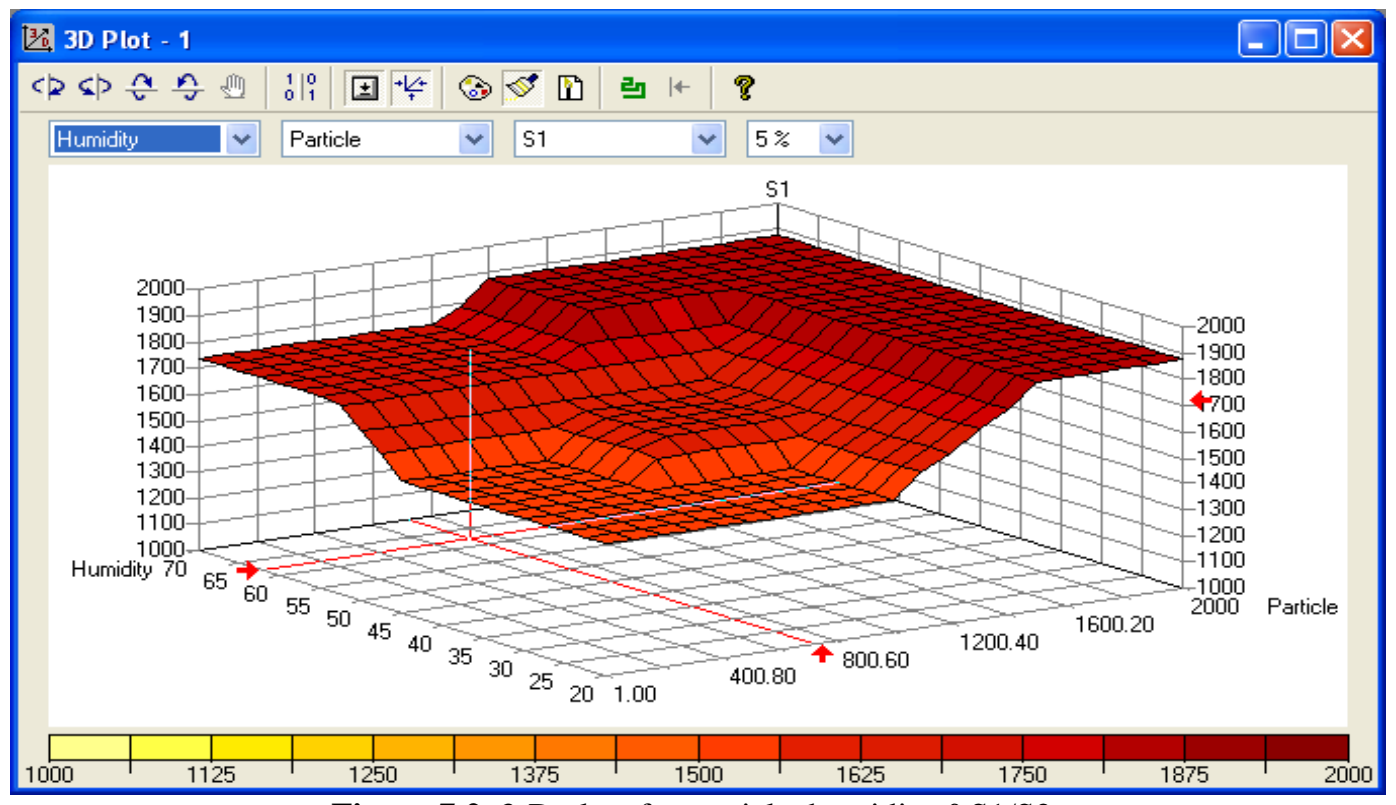

Figure 7.2. 3-D plots for particle, humidity $\& S 1 / \mathrm{S} 2$

The above figure plot shows that when the value of particle and humidity increases, the value of output parameter S1/S2 also raised quickly, according to the above fig. humidity will be countered as $63 \%$ and the value of particle is $698 \mathrm{ppm}$ then speed of S1/S2 will be $1735.3400 \mathrm{rpm}$. 


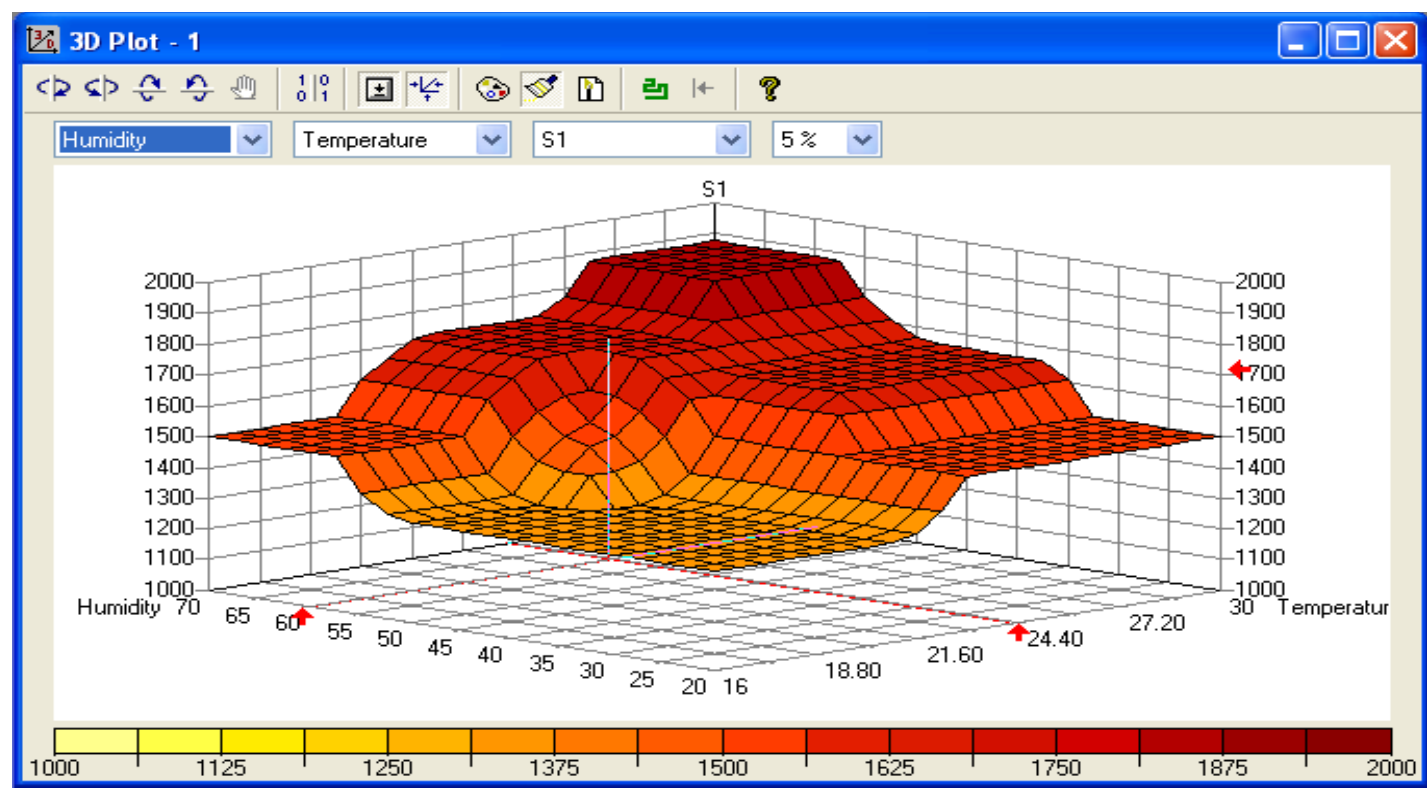

Figure 7.3. 3-D plots for temperature, humidity $\& S 1 / \mathrm{S} 2$

The above figure plot show that when the value of input parameter temperature and the humidity increases then the output parameter S1/S2 is also increasing. According to fig. when the value of humidity is at $63 \%$ and the value of temperature is $24.40 \%$ then speed of $\mathrm{S} 1 / \mathrm{S} 2$ will be $1729.0610 \mathrm{rpm}$.

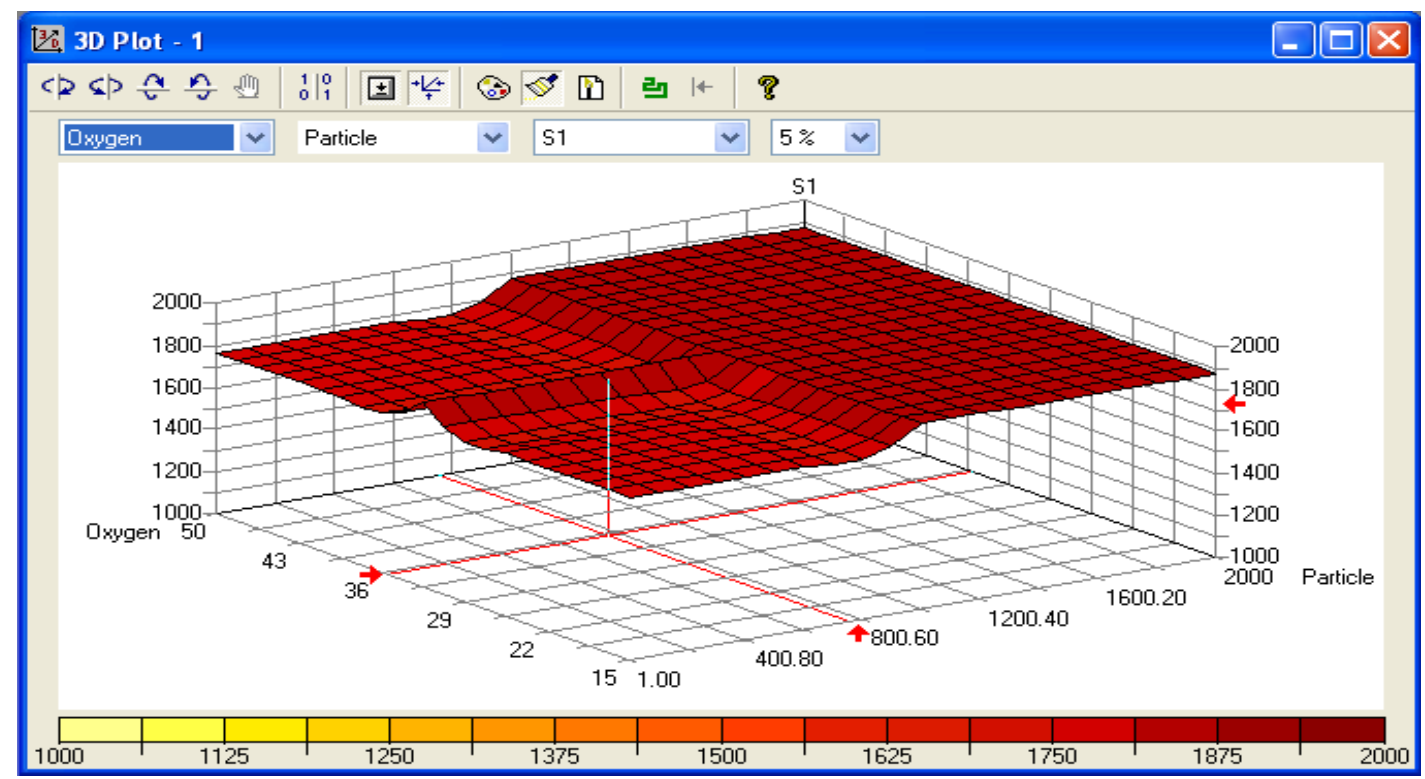

Figure 7.4. 3-D plots for oxygen, particle $\& S 1 / \mathrm{S} 2$

The above figure plot shows that when the value of input parameter oxygen and the particle increases then the output parameter S1/S2 is also increasing According to fig when the value of oxygen is $36 \%$ \& particle is $800 \mathrm{ppm}$ then speed of S1/S2 will be $1730.3800 \mathrm{rpm}$. 


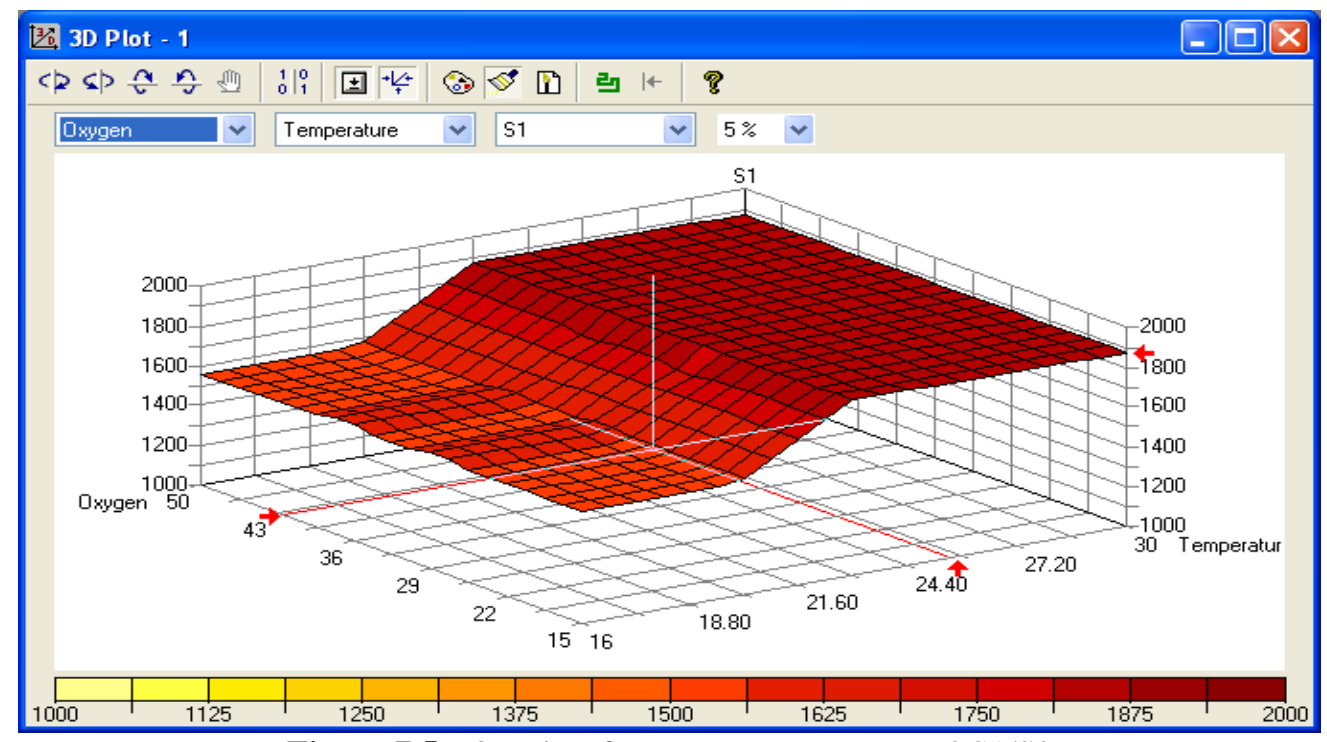

Figure 7.5. 3D plots for oxygen, temperature $\& S 1 / \mathrm{S} 2$

The above figure plot show that when the value of input parameter temperature and the oxygen increases then the output parameter S1/S2 is also increasing According to fig when the value of oxygen is $44 \%$ and the value of temperature is $25.90 \mathrm{C}$ then speed of $\mathrm{S} 1 / \mathrm{S} 2$ will be $1865.6000 \mathrm{rpm}$.

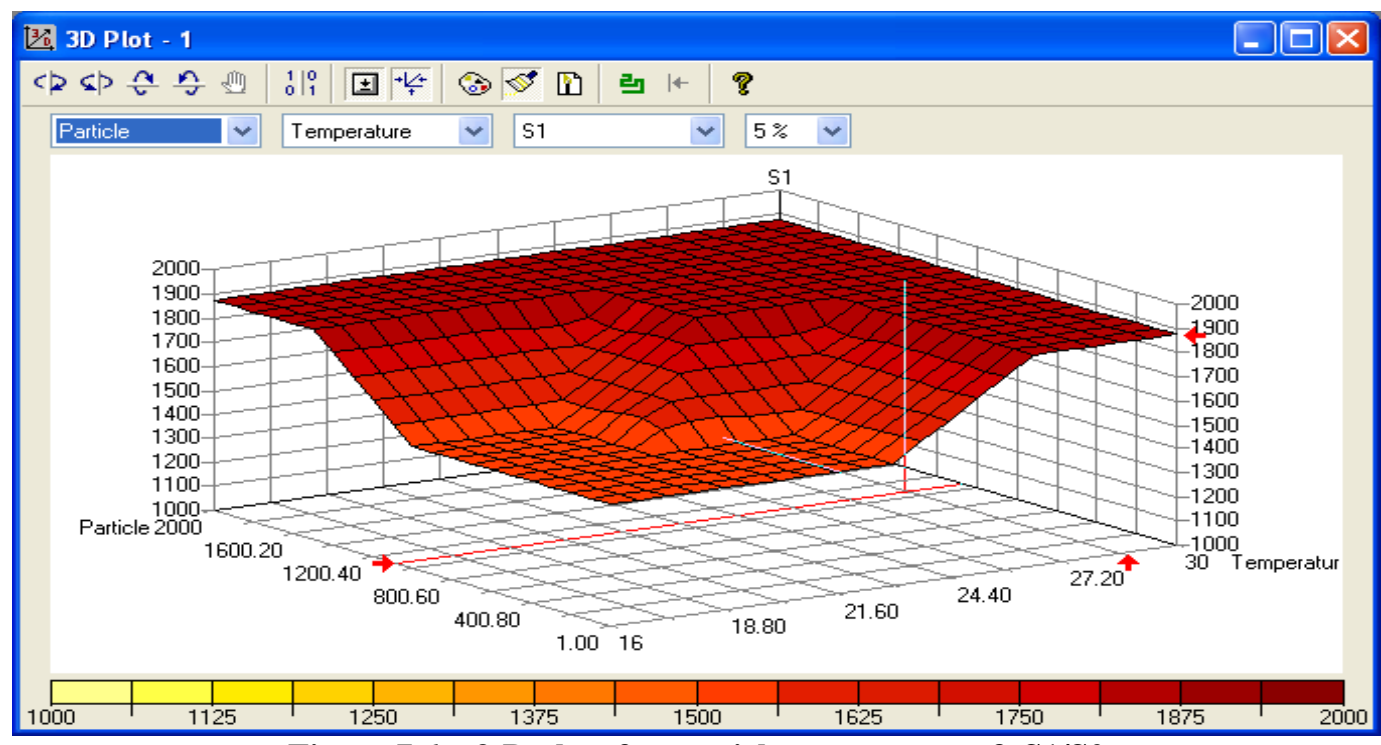

Figure 7.6 . 3-D plots for particle, temperature\& S1/S2

The above figure plot show that when the value of input parameter temperature and the particle increases then the output parameter S1/S2 is also increasing. According to fig when the value of particle are $910 \mathrm{ppm}$ and the value of temperature is $28.40 \mathrm{C}$ then speed of $\mathrm{S} 1 / \mathrm{S} 2$ will be $1865.0600 \mathrm{rpm}$.

\section{Conclusion \& Future Scope}

Fuzzy logic techniques are generally used for solving various problems where no definite model exists. The present work also fits into the same category because there are large numbers of parameters and their nonlinear interaction with each other is difficult to model. In the proposed research we have successfully designed a fuzzy logic based controller for environment control of operation theatre. This control approach using fuzzy logic is capable of controlling the nonlinearities of air conditioners and can stabilize the system quickly. All together the system assesses the value of temperature, amount of humidity, percentage of oxygen and amount of particles in air the system then adjust the speed as per the fuzzy rules applies on the speed of AC motors and Exhaust motor. Then, no abrupt difference will be seen on patient and their staff and economical also as it achieve optimum cooling. 
The fuzzy logic and artificial intelligence technique may be used to develop hybrid controller for the improvement in performance of the system. In future the fuzzy logic control of environment can be studied by improving the conditions of filtering system with reference to response time etc.

\section{References}

1. Febryan Hari Purwanto, Ema Utami, Eko Pramono, "Design of Server Room Temperature and Humidity Control System using Fuzzy Logic Based on Microcontroller" 2018 International Conference on Information and Communications Technology (ICOIACT).

2. Meisam Ramzanzad, Hamidreza Rashidy Kanan, "A New Method for Design and Implementation of Intelligent Traffic Control System Based on Fuzzy Logic Using FPGA” 2013 13th IEEE Iranian Conference on Fuzzy Systems (IFSC).

3. Zohaib Mushtaq, Akbare Yaqub, Muhammad Jabbar, Adnan Khalid, Saania Iqbal, Kamran Zeb, AbidA. Naqvi, "Environment Control System for Livestock Sheds using Fuzzy Logic Technique" 2016 3rd International Conference on Information Science and Control Engineering.

4. Hassen Taher Dorrah, Walaa Ibrahim Mahmoud Gabr, "New Approach for Automatic Control Modeling and Analysis Using Arithmetic and Visual Fuzzy Logic-based Representations in Fully Fuzzy Environment" Proceedings of the 2010 IEEE International Conference on Information and Automation June 20 - 23, Harbin, China

5. "Air conditioning requirements in hospitals" -by Lt Col (Dr) SKM Rao.

6. Güven K.O., "Hygienic air-conditioning in operating room”, 2013.

7. D.Driankov, H.Hellendoorn, M.Reinfrank. "An Introduction to Fuzzy Control."Narosa Publication.

8. Peker, T., "Standard, statutes of operating rooms,"Yayin/tesisat/ 73/7, Accessed April 2017.

9. "Modern refrigeration and air conditioning" -by Andrew, H. turnquist, bracciano.

10. "Handbook of air conditioning and refrigeration" Mcgraw hill Publication.

11. M.J.Patyra and D.M Mlynek. Fuzzy Logic Implementation and Applications. Wiley and Teubner, Chichester, NY, 1996.

12. Fuzzy TECH 5.5 User's Manual, 2001 INFORM GmbH/inform software corporation.

13. Timothy J.Ross.Fuzzy Logic with Engg. Applications. McGraw-Hill International Editions Electrical Engg. Series, 2015.

14. Malpica-Romero A. D, Assad G. D, "Fuzzy environmental control of surgical rooms in hospitals".

15. Rakesh Bhardwaj "PLC Based Automation of Heating, Ventilation And Air Conditioning (HVAC) System- Process Application".

16. Constantin Von Altrock. Fuzzy Logic and Neuro Fuzzy Applications Explained. PHI Private Ltd.

17. Agarwal, S., Joshi, A., Finin, T. and Yesha, Y., "A Pervasive Computing System for the Operating Room of the Future", http://ebiquity.umbc.edu/get/a/ publication/328.pdf, Accessed April 2006.

18. Orchard, B., "Fuzzy Sets", FuzzySet.html, Accessed April 2016.

19. Saritas Đ., Taskiran U., Unal Y., "Control of heat of operating room by approach of fuzzy method, 2005.

20. Tsoukalas L. H, Uhrig R. E, "Fuzzy and Neural Approaches in Engineering”, John Wiley \& Sons, Inc. New York, USA, 2017. 